\title{
A Comparison of Myocardial Perfusion Gated SPECT Studies Obtained at Rest and after Stress
}

\author{
Mahmoud A. Alfeeli ${ }^{a} \quad$ Ghulam M. Syed ${ }^{a}$ Henry W. Fielding ${ }^{a}$ Eman K. Alenizi $^{a}$ \\ Bert D. Collier ${ }^{a}$ Flemming Scheutz ${ }^{\mathrm{b}}$ \\ ${ }^{a}$ Department of Nuclear Medicine, Faculty of Medicine, and ${ }^{b}$ Department of Statistics, Faculty of Dentistry, \\ Kuwait University, Salmia, Kuwait
}

\section{Key Words}

Myocardial perfusion imaging $\cdot$ Gated single-photon emission tomography $\cdot$ Post-stress stunning

\begin{abstract}
Objective: To perform a comparison between post-stress and rest gated single-photon emission computer tomography (SPECT) myocardial perfusion imaging (MPI) studies to assess post-stress stunning and the variables underlying this phenomenon. Subjects and Methods: This was a prospective study of 318 consecutive adult patients undergoing

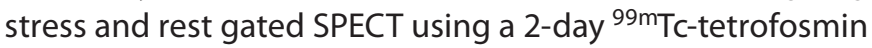
protocol. Bruce protocol treadmill stress $(n=93)$ or i.v. dipyridamole pharmacologic stress $(n=225)$ were used as stressors. Ejection fractions (EF) and left ventricular (LV) enddiastolic (EDV) and end-systolic volumes (ESV) were calculated using the Cedars Sinai Quantitative Gated SPECT software. Perfusion defects were visually scored using a 20-segment model to obtain summed stress scores. Statistical analysis was performed by applying paired $t$ test and multiregression. Statistically significant $(p<0.05)$ paired differences between post-stress and rest EF and ESV and type of stressing were noted. Results: Analysis of patient groups based on the type of stress showed significantly low EF on post-stress studies for both treadmill and dipyridamole stressing and also significantly high ESV on post-stress stud-
\end{abstract}

ies for dipyridamole. Multi-regression analysis using differences in post-stress and rest EF, EDV and ESV as dependent and summed stress scores, type of stress, delay time, previous myocardial infarction and size of LV as independent variables showed statistically significant associations between high summed stress scores ( $>13)$ and size of post-stress LV for EDV and ESV differences. High volumes were noted on post-stress studies. The magnitudes of the observed differences were well within the reproducibility of $L V$ volume calculations. Conclusions: Patients showing stunning had significant ischemia or a dilated LV. The stunning manifested as high EDV and ESV differences. The magnitude of the differences observed in EF and LV volumes were not clinically significant.

Copyright $\odot 2007$ S. Karger AG, Basel

\section{Introduction}

Myocardial perfusion imaging (MPI) studies are among the most common investigations performed for the diagnosis, evaluation of treatment response and prognosis of ischemic heart disease. Gated single-photon emission computer tomography (GSPECT) using ${ }^{99 \mathrm{~m} T c-}$ radiopharmaceuticals has become the procedure of choice for MPI since it provides functional and perfusion assessment simultaneously. The functional aspects of a

\section{KARGER \\ Fax +4161306 1234 \\ E-Mail karger@karger.ch}

www.karger.com
(C) 2007 S. Karger AG, Basel

1011-7571/07/0161-0034\$23.50/0

Accessible online at:

www.karger.com/mpp
Dr. Mahmoud Alfeeli

Department of Nuclear Medicine, Faculty of Medicine

PO Box 3003

Salmia 22031 (Kuwait)

Tel. +965 660 5625, Fax +965 533 8936, E-Mail malfeeli@hotmail.com 
GSPECT study derive from analysis of the myocardial wall motion at the time of imaging. By contrast, the perfusion study reflects the distribution of blood to the myocardium at the time of injection. All other factors being constant, rest and post-stress studies should ideally provide the same cardiac functional parameters in contrast to the perfusion assessment.

Some gated MPI studies have pointed to a phenomenon termed 'transient post-stress stunning'. This may lead to an underestimation of quantitative functional parameters from post-stress studies [1-4]. However, no firm conclusion has been drawn on post-stress stunning and its significance and implications for patient management. Similarly, factors which may be causing or contributing to this phenomenon, such as type of stress, extent of ischemia and previous myocardial infarction (MI), have not been investigated thoroughly.

The aims of this study were to compare post-stress and rest quantitative GSPECT functional parameters [ejection fraction (EF), end-diastolic volume (EDV) and endsystolic volume (ESV)] in a patient population referred to evaluate ischemic heart disease by GSPECT. Statistically significant lower cardiac function parameters on poststress study would support the transient stunning hypothesis and allow a further study into the impact of variables such as the severity of ischemic heart disease (as determined by perfusion defects), type of stress, previous episodes of MI, gender and age on the transient poststress stunning.

\section{Subjects and Methods}

This was a prospective consecutive case study. Each patient underwent a 2-day MPI protocol with stress imaging, usually followed by rest imaging. Our standard procedure was to first perform a stress study and, if normal, terminate the investigation. The study involved 318 patients. Of these, 93 were stressed by treadmill exercise according to the modified Bruce protocol, reaching at least $85 \%$ of the target heart rate (220 - age in years) or developing positive signs of ischemia (e.g. ECG changes, chest pain, blood pressure and heart rate changes), while 225 patients were stressed by i.v. infusion of dipyridamole $(0.142 \mathrm{mg} / \mathrm{kg} / \mathrm{min}$ while not exceeding $60 \mathrm{mg}$ in total). The mean age of the patients was $55 \pm 11$ years. A dose of $740 \mathrm{MBq}$ of ${ }^{99 \mathrm{~m}} \mathrm{Tc}$-tetrofosmin was injected at peak treadmill exercise and the stressing was terminated after a further $60 \mathrm{~s}$. For pharmacological stress the radiopharmaceutical was injected 2-3 min after completion of the infusion. For the rest study, each patient was also injected with a dose of $740 \mathrm{MBq}$ of ${ }^{99 \mathrm{~m}} \mathrm{Tc}$-tetrofosmin. The rest study was performed on average 6 days after the stress study.

Imaging was usually $60 \mathrm{~min}$ after injection of the radiopharmaceutical, using a dual-headed GE Millennium MG gamma camera (GEMS, Milwaukee, Oreg., USA). Patients were imaged in the supine position. Image acquisition was performed over 101 degrees of rotation, which provides 202 degrees of projection data, in 72 projection data sets. The projections were acquired into a $64 \times 64$ matrix, with a zoom of 1.33 and a $20 \%$ symmetrical energy window around $140 \mathrm{keV}$. Gating was performed into 8 bins per (RR) ECG cycle. The acquisition time per projection was $25 \mathrm{~s}$ and $15 \mathrm{~min}$ in total.

Reconstruction was performed using filtered back projection techniques on a GE Genie P/R workstation (GEMS). A Butterworth filter with a cutoff frequency of 0.43 and power of 7 was used for all studies. The QGS program from Cedars Sinai was used to calculate EDV, ESV and evaluation of myocardial wall motion. The left ventricular ejection fraction (LVEF) is calculated as EDV minus ESV divided by EDV. The validation of LVEF calculations by QGS has been reported by numerous authors [5-7]. The QGS software package is known to produce physiologically high EFs for small hearts [8] and 8-frame RR gating produces EFs that are $4-5$ percentage points lower than 16 -frame gating.

The evaluation for possible ventricular perfusion defects was performed following the method of Berman and colleagues [9, 10]: the myocardium is divided into 20 segments, with each segment being visually assigned a score from 0 to 4 , with 0 being normal. The summed stress score (SSS) is the sum of these 20 segment scores. Based upon previous prognostic work, the patients were divided into four SSS subgroups for evaluation of correlations between functional and perfusion deficits: $\mathrm{SSS}<4$ is considered normal while an SSS of 4-8 is considered mildly abnormal, an SSS of 9-13 moderately abnormal and an SSS $>13$ severely abnormal $[9,10]$.

For evaluation of ventricular dysfunction the SPSS software package (version 11.0; SPSS Inc., Chicago, Ill., USA) was used to study correlations amongst the variables. The differences between stress and rest values of EF, EDV and ESV were investigated using a paired $t$ test since the data consists of values from the same patient under two different situations (stress and rest). Multiple linear regression was used to study the effect of changes in the independent variables (age, SSS, history of previous MI, type of stress, LV dilatation, time between stress and rest studies and time from injection to imaging) on the dependent variables (EF, EDV and ESV).

\section{Results}

The mean delay time between the injection and start of imaging was $79 \pm 27 \mathrm{~min}$ in stress and $83 \pm 34 \mathrm{~min}$ in rest studies, the difference between these times was not statistically significant (paired t test: $t=-1.56, \mathrm{p}=0.12$ ).

Of the 318 cases studied, the pattern of myocardial perfusion was normal on both stress and rest images in 93, showed completely reversible perfusion defects in 115 patients, partially reversible perfusion defects in 61, mixed reversible and fixed perfusion defects in 17 and fixed perfusion defects or apparent scars in 32. 
Table 1. Means, SD, paired t test $\mathrm{p}$ value, and number of patients for EF in type of stress against SSS

\begin{tabular}{|c|c|c|c|c|c|}
\hline \multirow[t]{2}{*}{ Groups } & \multicolumn{5}{|l|}{ SSS } \\
\hline & all & $<4$ & $4-8$ & $9-13$ & $>13$ \\
\hline \multicolumn{6}{|l|}{ Total } \\
\hline Stress & $56 \pm 14$ & $62 \pm 10$ & $58 \pm 12$ & $46 \pm 13$ & $39 \pm 14$ \\
\hline Rest & $57 \pm 14$ & $64 \pm 11$ & $59 \pm 11$ & $48 \pm 13$ & $41 \pm 14$ \\
\hline $\mathrm{p}$ value & $0.000^{* *}$ & $0.007^{* *}$ & 0.124 & 0.053 & 0.059 \\
\hline $\mathrm{n}$ & 318 & 157 & 68 & 59 & 34 \\
\hline \multicolumn{6}{|c|}{ Dipyridamole } \\
\hline Stress & $55 \pm 15$ & $63 \pm 11$ & $58 \pm 12$ & $45 \pm 12$ & $39 \pm 15$ \\
\hline Rest & $57 \pm 15$ & $64 \pm 11$ & $60 \pm 10$ & $47 \pm 13$ & $41 \pm 15$ \\
\hline $\mathrm{p}$ value & $0.001^{* *}$ & $0.034^{*}$ & 0.119 & 0.168 & 0.185 \\
\hline $\mathrm{n}$ & 225 & 103 & 49 & 44 & 29 \\
\hline \multicolumn{6}{|l|}{ Treadmill } \\
\hline Stress & $57 \pm 12$ & $61 \pm 10$ & $57 \pm 12$ & $47 \pm 14$ & $39 \pm 5$ \\
\hline Rest & $59 \pm 13$ & $63 \pm 11$ & $57 \pm 13$ & $50 \pm 15$ & $43 \pm 5$ \\
\hline $\mathrm{p}$ value & $0.018^{*}$ & 0.087 & 0.774 & 0.156 & $0.005^{* *}$ \\
\hline n & 93 & 54 & 19 & 15 & 5 \\
\hline
\end{tabular}

* Differences were significant $(\mathrm{p}<0.05)$ between post-stress and corresponding rest parameter in the same group.

** Differences were highly significant $(\mathrm{p}<0.01)$ between post-stress and corresponding rest parameter in the same group.
The mean \pm SD for both post-stress and rest studies in all patients, along with the subgroups based on the method of stressing, are shown for EF in table 1, EDV in table 2 and ESV in table 3 . The paired differences between post-stress and rest EFs were statistically significant based on Student's paired t test for both stressing types. The paired ESV differences were statistically significant for the total and dipyridamole populations. The EF was high on rest studies and the ESV was large on post-stress studies.

In the SSS subgroups, the paired differences between rest and post-stress EFs and EDVs were significantly high in the rest studies for the SSS $<4$ group for both the total and dipyridamole stressing populations. In the group with SSS $>13$, the EDV and ESV values were high for poststress studies, and the ESV and EDV paired differences were highly significant for both the total and dipyridamole stressing populations.

The multiple linear regression analysis results are summarized in table 4 . The SSS $>13$ subgroup showed statistically significant differences for EDV and ESV. A significant association with LV size on post-stress studies (dilated or normal size) was observed. Other significant paired differences previously noted in SSS subgroups based on type of stressing were not seen in this multiple regression analysis.

\section{Discussion}

Transient post-stress stunning during MPI has been described in recent years [11-13]; however, factors playing a role in this phenomenon and the implications are not well understood. Transient stunning has been attributed to stress-induced ischemia [11], circulating levels of catecholeamine [1], previous MI [14] and to the pharmacologic effects of dipyridamole [1]. Transient ischemic dilatation of the LV, despite being well documented, has no definite explanation. Possible explanations for transient ischemic dilatation include subendocardial hypoperfusion, systolic LV dysfunction and even actual physical dilatation with ischemia $[3,15,16]$. Both stunning and transient ischemic dilatation are associated with cardiac stress and ischemia and may impact on wall motion and quantitative measures as reported $[3,15,16]$. These phenomena would result in post-stress parameters being less reliable than rest measures for LV function and wall motion.

A 2-day protocol is preferable in investigating poststress stunning since the duration of stunning is unknown. Some studies have suggested that it lasts for less than $1 \mathrm{~h}$ while others say it continues for days [1].

The means of the functional LV parameters on poststress GSPECT, i.e. EF, EDV and ESV in all patients, sub- 
Table 2. Means, SD, paired t test $\mathrm{p}$ value, and number of patients for EDV (ml) in type of stress against SSS
Table 3. Means, SD, paired t test p value, and number of patients for ESV $(\mathrm{ml})$ in type of stress against SSS

\begin{tabular}{|c|c|c|c|c|c|}
\hline \multirow[t]{2}{*}{ Groups } & \multicolumn{5}{|l|}{ SSS } \\
\hline & all & $<4$ & $4-8$ & $9-13$ & $>13$ \\
\hline \multicolumn{6}{|l|}{ All } \\
\hline Stress & $93 \pm 45$ & $77 \pm 27$ & $84 \pm 26$ & $113 \pm 48$ & $150 \pm 70$ \\
\hline Rest & $93 \pm 42$ & $79 \pm 27$ & $85 \pm 25$ & $112 \pm 47$ & $143 \pm 65$ \\
\hline $\mathrm{p}$ value & 0.428 & $0.001^{* *}$ & 0.407 & 0.558 & $0.023^{*}$ \\
\hline $\mathrm{n}$ & 318 & 157 & 68 & 59 & 34 \\
\hline \multicolumn{6}{|c|}{ Dipyridamole } \\
\hline Stress & $94 \pm 48$ & $75 \pm 27$ & $80 \pm 22$ & $115 \pm 47$ & $153 \pm 75$ \\
\hline Rest & $94 \pm 44$ & $77 \pm 26$ & $81 \pm 20$ & $114 \pm 45$ & $143 \pm 70$ \\
\hline $\mathrm{p}$ value & 0.830 & $0.011^{*}$ & 0.587 & 0.500 & $0.004^{* *}$ \\
\hline $\mathrm{n}$ & 225 & 103 & 49 & 44 & 29 \\
\hline \multicolumn{6}{|l|}{ Treadmill } \\
\hline Stress & $90 \pm 36$ & $81 \pm 28$ & $92 \pm 33$ & $106 \pm 51$ & $135 \pm 31$ \\
\hline Rest & $92 \pm 37$ & $84 \pm 28$ & $94 \pm 35$ & $105 \pm 63$ & $144 \pm 29$ \\
\hline $\mathrm{p}$ value & 0.052 & 0.056 & 0.458 & 0.922 & 0.182 \\
\hline $\mathrm{n}$ & 93 & 54 & 19 & 15 & 5 \\
\hline
\end{tabular}

* Differences were significant $(\mathrm{p}<0.05)$ between post-stress and corresponding rest parameter in the same group.

** Differences were highly significant $(\mathrm{p}<0.01)$ between post-stress and corresponding rest parameter in the same group.

\begin{tabular}{|c|c|c|c|c|c|}
\hline \multirow[t]{2}{*}{ Groups } & \multicolumn{5}{|l|}{ SSS } \\
\hline & all & $<4$ & $4-8$ & $9-13$ & $>13$ \\
\hline \multicolumn{6}{|l|}{ All } \\
\hline Stress & $46 \pm 38$ & $31 \pm 20$ & $37 \pm 19$ & $66 \pm 40$ & $98 \pm 62$ \\
\hline Rest & $45 \pm 37$ & $31 \pm 20$ & $37 \pm 20$ & $63 \pm 41$ & $91 \pm 59$ \\
\hline $\mathrm{p}$ value & $0.019^{*}$ & 0.936 & 0.882 & 0.121 & $0.005^{* *}$ \\
\hline $\mathrm{n}$ & 318 & 157 & 68 & 59 & 34 \\
\hline \multicolumn{6}{|c|}{ Dipyridamole } \\
\hline Stress & $47 \pm 41$ & $30 \pm 19$ & $35 \pm 16$ & $67 \pm 39$ & $101 \pm 66$ \\
\hline Rest & $46 \pm 39$ & $30 \pm 19$ & $34 \pm 15$ & $65 \pm 39$ & $93 \pm 63$ \\
\hline $\mathrm{p}$ value & $0.009^{* *}$ & 0.913 & 0.429 & 0.155 & $0.005^{* *}$ \\
\hline $\mathrm{n}$ & 225 & 103 & 49 & 44 & 29 \\
\hline \multicolumn{6}{|l|}{ Treadmill } \\
\hline Stress & $43 \pm 30$ & $34 \pm 21$ & $43 \pm 26$ & $61 \pm 42$ & $84 \pm 25$ \\
\hline Rest & $42 \pm 31$ & $33 \pm 21$ & $44 \pm 28$ & $59 \pm 47$ & $83 \pm 23$ \\
\hline $\mathrm{p}$ value & 0.789 & 0.752 & 0.483 & 0.502 & 0.888 \\
\hline $\mathrm{n}$ & 93 & 54 & 19 & 15 & 5 \\
\hline
\end{tabular}

* Differences were significant $(\mathrm{p}<0.05)$ between post-stress and corresponding rest parameter in the same group.

** Differences were highly significant $(\mathrm{p}<0.01)$ between post-stress and corresponding rest parameter in the same group. 
Table 4. Results of the multiple linear regressions in $p$ values

\begin{tabular}{|c|c|c|c|}
\hline \multirow[t]{2}{*}{ Parameter } & \multicolumn{3}{|c|}{ Differences } \\
\hline & $\mathrm{EF}$ & EDV & ESV \\
\hline \multicolumn{4}{|l|}{ Type of stress } \\
\hline $\begin{array}{l}\text { (treadmill vs. } \\
\text { dipyridamole) }\end{array}$ & 0.746 & 0.108 & 0.433 \\
\hline $\begin{array}{l}\text { History of previous MI } \\
\text { SSS }\end{array}$ & 0.881 & 0.477 & 0.813 \\
\hline$<4(\mathrm{n}=157)$ & - & - & - \\
\hline $4-8(n=68)$ & 0.800 & 0.541 & 0.948 \\
\hline $9-13(n=59)$ & 0.634 & 0.703 & 0.736 \\
\hline$>13(\mathrm{n}=34)$ & 0.481 & $0.015^{*}$ & $0.021^{*}$ \\
\hline LV dilatation & 0.571 & $0.007^{* *}$ & $0.043^{*}$ \\
\hline Age & 0.537 & 0.984 & 0.464 \\
\hline \multicolumn{4}{|c|}{ Difference in days between stress and rest } \\
\hline$\leq 1(\mathrm{n}=25)$ & - & - & - \\
\hline $1-2(n=29)$ & 0.274 & 0.903 & 0.421 \\
\hline $3-5(n=66)$ & 0.111 & 0.095 & 0.095 \\
\hline $6-7(n=150)$ & 0.570 & 0.095 & 0.213 \\
\hline$>7(\mathrm{n}=48)$ & 0.476 & 0.303 & 0.594 \\
\hline \multicolumn{4}{|c|}{ Time between stress injection and imaging, $\mathrm{h}$} \\
\hline$<1(\mathrm{n}=87)$ & - & - & - \\
\hline $1-1.5(\mathrm{n}=141)$ & 0.499 & 0.713 & 0.669 \\
\hline $1.5-2(n=64)$ & 0.146 & 0.917 & 0.342 \\
\hline$>2(\mathrm{n}=26)$ & 0.154 & 0.364 & 0.387 \\
\hline
\end{tabular}

* Differences were significant $(\mathrm{p}<0.05)$ between post-stress and corresponding rest parameter in the same group.

** Differences were highly significant $(\mathrm{p}<0.01)$ between poststress and corresponding rest parameter in the same group.

groups based on SSS and on stress type are comparable to those previously reported $[17,18]$. This suggests that any difference in our methodology and population had no significant effect. The expected trend of smaller EF and LV dilation with increasing SSS score was observed for all patient populations.

The paired t test results show significance for EF differences in both types of stressing while ESV changes are seen only in the dipyridamole group. The dipyridamole stress group shows significant differences in EDV and ESV only in the SSS $<4$ and SSS $>13$ subgroups. The treadmill stress group shows no significant differences in either EDV or ESV. Consequently, stunning phenomena may manifest differently in the same type of stress due to a different physiological response.

The findings of this study suggest that the subgroup of patients who have significant reversible ischemia will manifest LV dysfunction on post-stress MPI and GSPECT studies. These patients show marginal improvement on rest studies. However, the reproducibility in calculating EF from ventricular volumes for patients is $5 \%$ and can thus account for these marginal improvements [19]. The EF paired differences have a $95 \%$ confidence interval of -28 to 24 ; thus we conclude that although pairing tests give statistically significant differences, these were clinically inconsequential in this study - in contrast to previously reported stunning phenomena results [11-13].

Transient LV dysfunction has diagnostic and prognostic utility [20]. It has been shown that this finding is associated with severe and extensive coronary artery disease. Some studies have even reported a higher specificity of this finding in the diagnosis of coronary artery disease than in multiple perfusion defects $[21,22]$. The LV dilatation ratio has been found to be a reliable quantitative indicator of the severity of coronary artery disease [20]. Another study showed that the overall prognosis for patients with transitory LV changes on post-stress study is worse, as they show a high rate of cardiac events and mortality [23]. This study demonstrated a relationship between transient ischemic dilatation, severe perfusion defects and transient stunning. Our results indicate that transient stunning is clinically insignificant for this patient population and imaging protocol.

\section{Conclusion}

This study demonstrated post-stress stunning in an SSS subgroup of patients with severe ischemia and established an association between LV dysfunction and LV dilatation on post-stress studies. This finding was observed in all stress type subgroups. As many factors probably play a role in this phenomenon, a detailed analysis of variables by applying multiple linear regression is preferable in such studies. The observed stunning was not clinically significant in this study.

\section{Acknowledgment}

Work supported in part by a fund from the College of Graduate Studies, Kuwait University, Kuwait. 


\section{References}

$\checkmark 1$ Lee DS, Yeo JS, Chung JK, Lee MM, Lee MC: Transient prolonged stunning induced by dipyridamole and shown on 1- and 24-hour poststress 99mTc-MIBI gated SPECT. J Nucl Med 2000;41:27-35.

$>2$ Hashimoto J, Kubo A, Iwasaki R, Iwanaga S, Mitamura H, Ogawa S, Kosuda S: Gated single photon emission tomography imaging protocol to evaluate myocardial stunning after exercise. Eur J Nucl Med 1999;26:15411546.

$>3$ Bestetti A, Di Leo C, Alessi A, Triulzi A, Tagliabue L, Tarolo GL: Post-stress end-systolic left ventricular dilatation: a marker of endocardial post-ischemic stunning. Nucl Med Commun 2001;22:685-693.

$>4$ Paeng JC, Lee DS, Yeo JS, Noh CI, Kim YK, Chung JK, Lee MC: Septal stunning by dipyridamole stress shown on quantitative gated perfusion SPECT in a child with hypertrophic cardiomyopathy. Clin Nucl Med 2002;27:96-100.

5 Germano G, Kiat H, Kavanagh PB, Moriel M, Mazzanti M, Su HT, Van Train KF, Berman DS: Automatic quantification of ejection fraction from gated myocardial perfusion SPECT. J Nucl Med 1995;36:2138-2147.

$\checkmark 6$ Kumita S, Cho K, Nakajo H, Toba M, Uwamori M, Mizumura S, Kumazaki T, Sano J, Sakai S, Munakata K: Assessment of left ventricular diastolic function with electrocardiography-gated myocardial perfusion SPECT: comparison with multigated equilibrium radionuclide angiography. J Nucl Cardiol 2001;8:568-574.

7 Faber TL, Vansant JP, Pettigrew RI, Galt JR, Blais M, Chatzimavroudis G, Cooke CD, Folks RD, Waldrop SM, Gurtler-Krawczynska E, Wittry MD, Garcia EV: Evaluation of left ventricular endocardial volumes and ejection fractions computed from gated perfusion SPECT with magnetic resonance imaging: comparison of two methods. J Nucl Cardiol 2001;8:645-651.

$>8$ Nakajima K, Taki J, Higuchi T, Kawano M, Taniguchi M, Maruhashi K, Sakazume S, Tonami N: Gated SPET quantification of small hearts: mathematical simulation and clinical application. Eur J Nucl Med 2000;27: 1372-1379. $\checkmark 9$ Berman DS, Hachamovitch R, Kiat H, Cohen I, Cabico JA, Wang FP, Friedman JD, Germano G, Van Train K, Diamond GA: Incremental value of prognostic testing in patients with known or suspected ischemic heart disease: a basis for optimal utilization of exercise technetium-99m sestamibi myocardial perfusion single-photon emission computed tomography. J Am Coll Cardiol 1995;26:639-647.

10 Hachamovitch R, Berman DS, Kiat H, Cohen I, Lewin $\mathrm{H}$, Amanullah A, Kang X, Friedman J, Diamond GA: Incremental prognostic value of myocardial perfusion single photon emission computed tomography and impact on subsequent management in patients with or suspected of having myocardial ischemia. Am J Cardiol 1997;80:426433.

11 Borges-Neto S, Javaid A, Shaw LK, Kong DF, Hanson MW, Pagnanelli RA, Ravizzini G, Coleman RE: Poststress measurements of left ventricular function with gated perfusion SPECT: comparison with resting measurements by using a same-day perfusionfunction protocol. Radiology 2000;215: 529-533.

12 Berman DS, Germano G: Evaluation of ventricular ejection fraction, wall motion, wall thickening, and other parameters with gated myocardial perfusion single-photon emission computed tomography. J Nucl Cardiol 1997;4:S169-S171.

$>13$ Chua T, Kiat H, Germano G, Maurer G, van Train K, Friedman J, Berman D: Gated technetium-99m sestamibi for simultaneous assessment of stress myocardial perfusion, postexercise regional ventricular function and myocardial viability. Correlation with echocardiography and rest thallium-201 scintigraphy. J Am Coll Cardiol 1994;23: 1107-1114.

14 Hambye AS, Van Den Branden F, Vandevivere I: Diagnostic value of Tc-99m sestamibi gated SPECT to assess viability in a patient after acute myocardial infarction. Clin Nucl Med 1996;21:19-23.

15 Marcassa C, Galli M, Baroffio C, Campinini R, Giannuzzi P: Transient left ventricular dilation at quantitative stress-rest sestamibi tomography: clinical, electrocardiographic and angiographic correlates. J Nucl Cardiol 1999;6:397-405.
16 Van Tosh A, Hecht S, Berger M, Roberti R, Luna E, Horowitz SF: Exercise echocardiographic correlates of transient dilatation of the left ventricular cavity on exercise thallium-201 SPECT imaging. Chest 1994;106: 1725-1729.

17 De Bondt P, Van de Wiele C, De Sutter J, De Winter F, De Backer G, Dierckx RA: Ageand gender-specific differences in left ventricular cardiac function and volumes determined by gated SPECT. Eur J Nucl Med 2001; 28:620-624.

18 Ababneh AA, Sciacca RR, Kim B, Bergmann SR: Normal limits for left ventricular ejection fraction and volumes estimated with gated myocardial perfusion imaging in patients with normal exercise test results: influence of tracer, gender and acquisition camera. J Nucl Cardiol 2000;7:661-668.

19 Johnson LL, Verdesca SA, Aude WY, Xavier RC, Nott LT, Campanella MW, Germano G: Post-ischemic stunning can affect left ventricular ejection fraction and regional wall motion on post-stress gated sestamibi tomograms. J Am Coll Cardiol 1997;30:16411648.

20 McLaughlin MG, Danias PG: Transient ischemic dilation: a powerful diagnostic and prognostic finding of stress myocardial perfusion imaging. J Nucl Cardiol 2002;9:663667.

21 Chouraqui P, Rodrigues EA, Berman DS, Maddahi J: Significance of dipyridamole-induced transient dilation of the left ventricle during thallium-201 scintigraphy in suspected coronary artery disease. Am J Cardiol 1990;66:689-694.

22 Takeishi Y, Chiba J, Abe S, Komatani A, Takahashi A, Tomoiki H: Noninvasive identification of the left main and three-vessel coronary artery disease by thallium-201 single photon emission computed tomography during adenosine infusion. Ann Nucl Med 1994;8:1-7.

23 McClellan JR, Travin MI, Herman SD, Baron JI, Golub RJ, Gallagher JJ, Waters D, Heller GV: Prognostic importance of scintigraphic left ventricular cavity dilation during intravenous dipyridamole technetium-99m sestamibi myocardial tomographic imaging in predicting coronary events. Am Coll Cardiol 1997;79:600-605. 